\title{
Retracted: Electrical conductivity of hydrogenated armchair nanoribbon as a gas sensor using non-equilibrium Green's function method
}

\author{
Rostam Moradian ${ }^{1,2,3}$ and Sahar Nazeri ${ }^{1,2^{*}}$
}

\begin{abstract}
Retraction
This article was mistakenly published twice. For this reason this duplicate article has now been retracted. For citation purposes please cite the original: http://www.inljournal.com/?_action=articlelnfo\&article=16

Abstract

Nanosensing properties of hydrogenated edge armchair graphene nanoribbons (HAGNR) are investigated. Using non-equilibrium Green's function method in the tight-binding approach, the effects of hydrogen and oxygen adsorption on current-voltage (I-V) characteristics and also the electrical conductivity of these systems are calculated. We found that the $I-V$ curves of these systems change by the adsorption of hydrogen or oxygen molecules. Also, we found that conductivity of these systems at low adsorption concentrations increases, while at high adsorption, concentrations decrease. This could be explained in terms of semiconducting or metallic properties of the adsorbed system which was obtained from electronic properties of our clean HAGNR system. On the other hand, the local density of states of some sites has a metallic behavior, and that of other sites has a semiconducting behavior.

Note that our results are investigated at a fixed temperature $T=300 \mathrm{~K}$, i.e., room temperature. By calibrating conductivity in terms of adsorbed gas molecules, one can make a gas nanosensor.
\end{abstract}

Keywords: nanosensor, graphene nanoribbon, gas adsorption, electrical conductivity, non-equilibrium Green's function

\section{Background}

In recent years, much attention has been focused on nanoelectronic devices [1-3] and molecular gas adsorption on single-walled carbon nanotubes and graphene system surfaces [4-13]. Since gas adsorption by these systems could change their electronic properties, these properties could be used as a gas sensing property. Electronic and transport properties of these systems such as local density of states (LDOS), transmission coefficient, and currentvoltage $(I-V)$ characteristics are investigated by the Landaure formula and non-equilibrium Green's function (NEGF) formalism [14-19].

\footnotetext{
* Correspondence: nazeri.sahar@gmail.com

${ }^{1}$ Physics Department, Faculty of Science, Razi University, Kermanshah 6714967346, Iran

${ }^{2}$ Nano Technology Research Laboratory, Razi University, Kermanshah 6714967346, Iran

Full list of author information is available at the end of the article
}

One of the various forms of these devices that have attracted a great deal of attention is graphene nanoribbons. The electronic properties of nanoribbons could be determined by the atomic geometry along their edges, namely, zigzag graphene nanoribbons (ZGNR) or armchair graphene nanoribbons (AGNR) [14,20-23]. Usually, ZGNRs have a metallic feature, while AGNRs are semiconducting [14].

Here, we extend our previous work [14] to investigate the gas sensing properties of these systems. We focus on the AGNRs which were hydrogenated from their edges, called hydrogenated AGNR (HAGNR). Note that, in the previous work, we considered a clean AGNR and ZGNR without adsorption of hydrogen or oxygen molecules, but in this paper, we considered a HAGNR where hydrogen or oxygen molecules were adsorbed on its surface. Our results show that at low adsorption concentrations, more adsorption of hydrogen or oxygen 


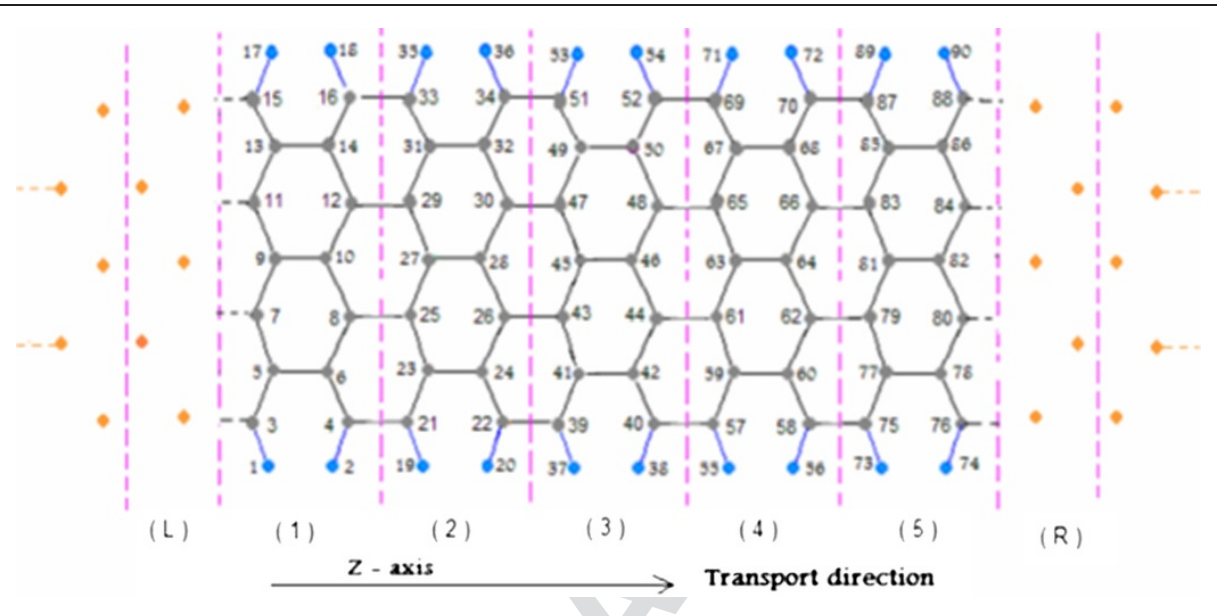

Figure 1 A hydrogenated edge armchair, HAGNR (device), attached to the left (L) and right (R) leads. The device is divided into similar cells where each cell included 14 carbon atoms and 4 hydrogen atoms. The blue points are hydrogen atoms.

molecules leads to the increase of conductivity of the system, while at high adsorption concentrations, more adsorption leads to the reduction of conductivity. Therefore, conductivity could identify the percentage of adsorbed gas molecules.

This paper is organized as follows: In the 'Methods' section, we describe the model and theoretical tools that we used to calculate the local density of states, transmission, and current-voltage curves of the system. In the 'Results and discussion' section, we applied this method to our system and analyzed the obtained results. The last section is the conclusion part.

\section{Method}

Consider a HAGNR which is attached to two leads from the left to the right. The HAGNR is called the device. To convert this system to a one-dimensional system, we divided it into similar cells along the transport direction where each cell included $N_{0}$ atoms (each lattice site
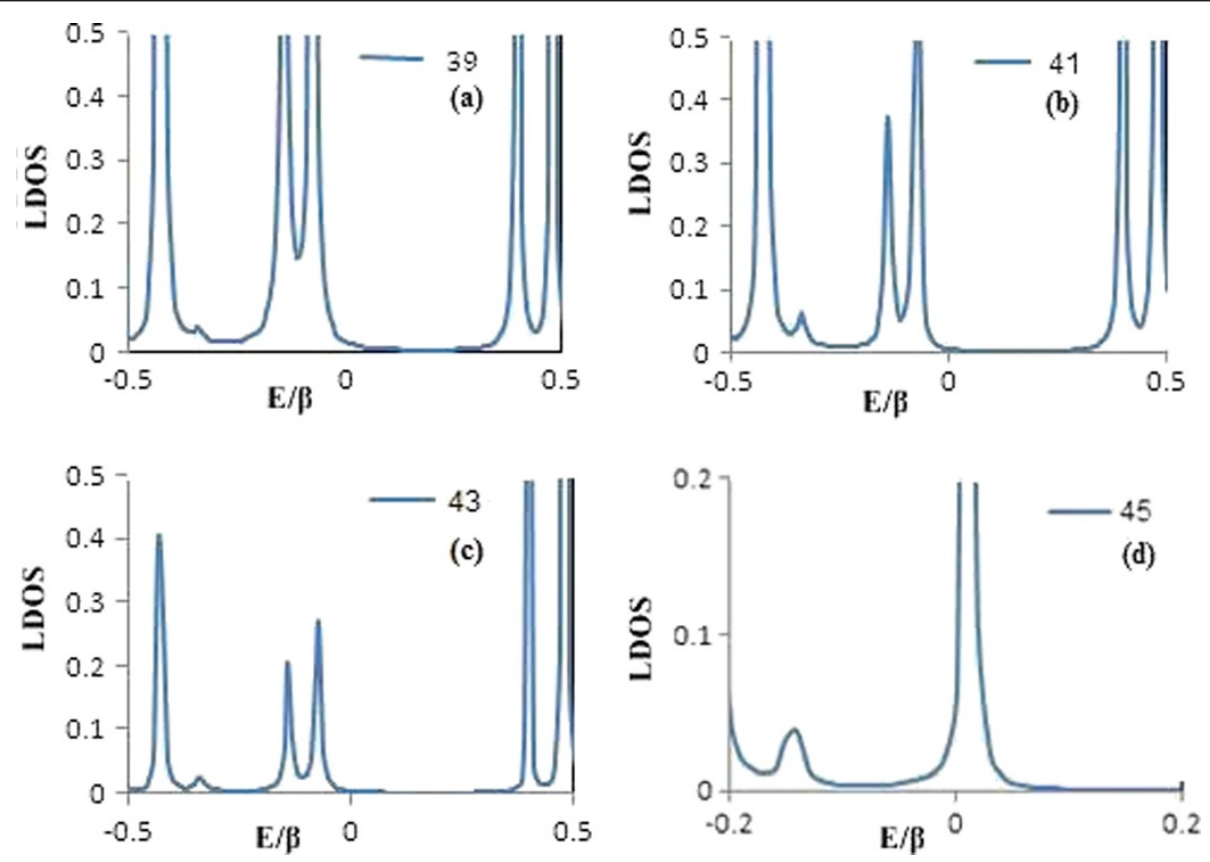

Figure 2 Comparison of LDOS of sites $\mathbf{3 9}, \mathbf{4 1}, \mathbf{4 3}$, and $\mathbf{4 5}$ along the width of clean HAGNR. (a) LDOS of site 39, (b) LDOS of site 41, (c) LDOS of site 43 , and (d) LDOS of site 45 . 


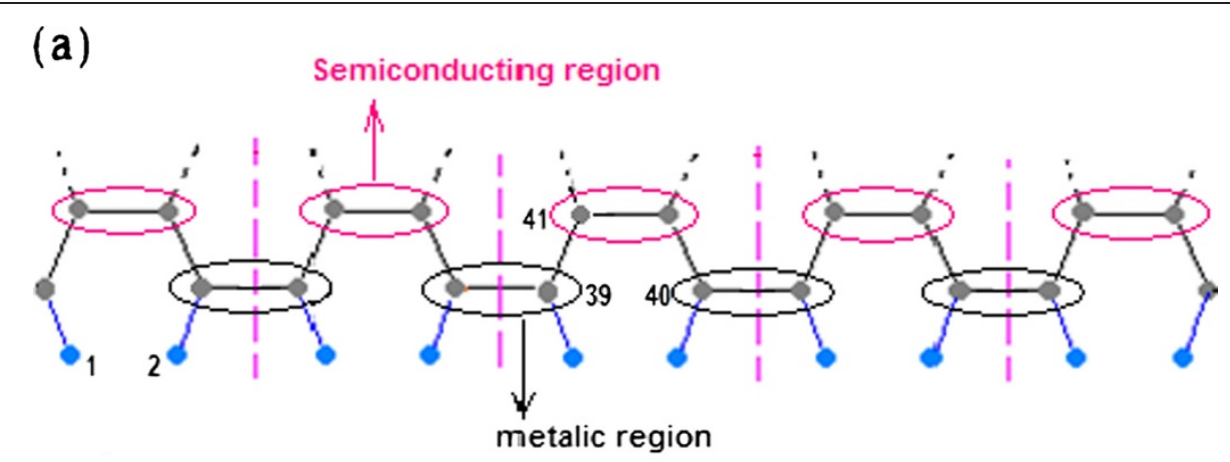

(b)

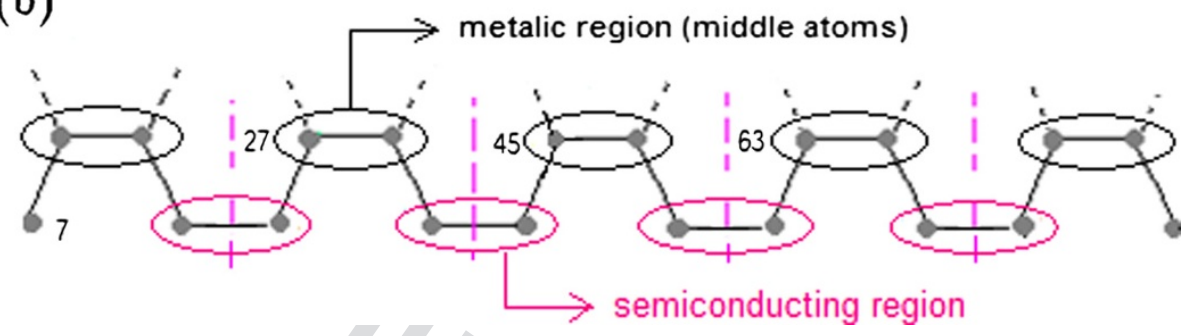

Figure 3 Metallic and semiconducting regions for (a) lower edge nanowire and (b) middle nanowire.

included $N_{0}$ sublattice atoms). This is illustrated in Figure 1.

In the NEGF formalism, in the absence of applied voltage, the total transmission is given by $[14,16]$,

$$
T=4 \operatorname{Tr}\left[\operatorname{Im}\left(\Sigma_{R}\right) G_{N+1,0}^{r} \operatorname{Im}\left(\Sigma_{L}\right) G_{0, N+1}^{a}\right],
$$

where $\operatorname{Im}[x]$ stands for the imaginary part of $x$, and $G_{i i^{\prime}}^{\mathrm{r}}\left(G_{i i^{\prime}}^{\mathrm{a}}\right)$ is the retarded (advance) Green function matrix from $i$ unit cell to $i^{\prime}$. The unit cell number $i=0$ refers to the right edge of the left lead, and $i=N+1$ refers to the left edge of right lead. Note that we have $N$ unit cells in the device region $(i=1, \ldots, N)$ and that in our system, $N$ is equal to 5 . The retarded and advance Green functions, $G^{\mathrm{r}}$ and $G^{\mathrm{a}}$, are defined by

$$
G^{r}[(E+i \eta) S-H]=I
$$

and

$$
G^{\mathrm{a}}=\left[G^{\mathrm{a}}(E)\right]^{\dagger},
$$

respectively, where $S$ and $I$ are the overlap matrix and unitary matrix, respectively. $H$ is the Hamiltonian (a)

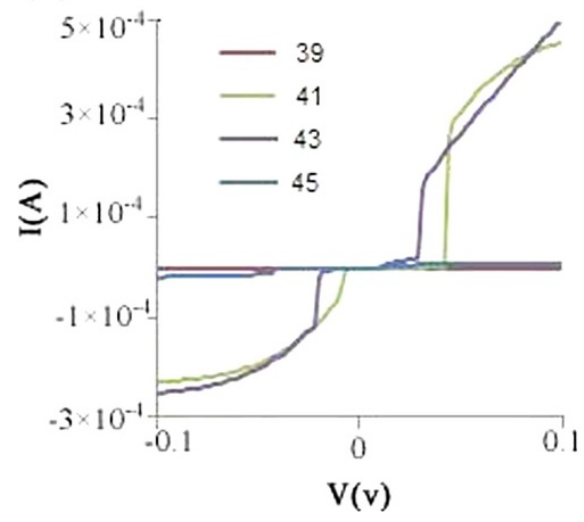

(b)

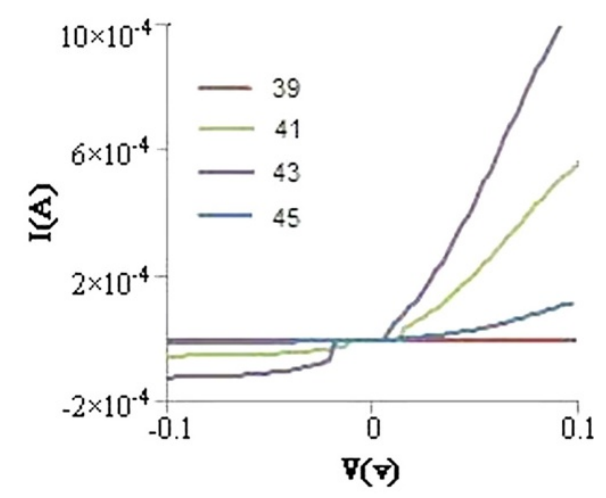

Figure 4 Current-voltage characteristics. I-V characteristics of our system when (a) one hydrogen molecule or (b) one oxygen molecule is adsorbed by sites $39,41,43$, and 45 along the width of HAGNR. 

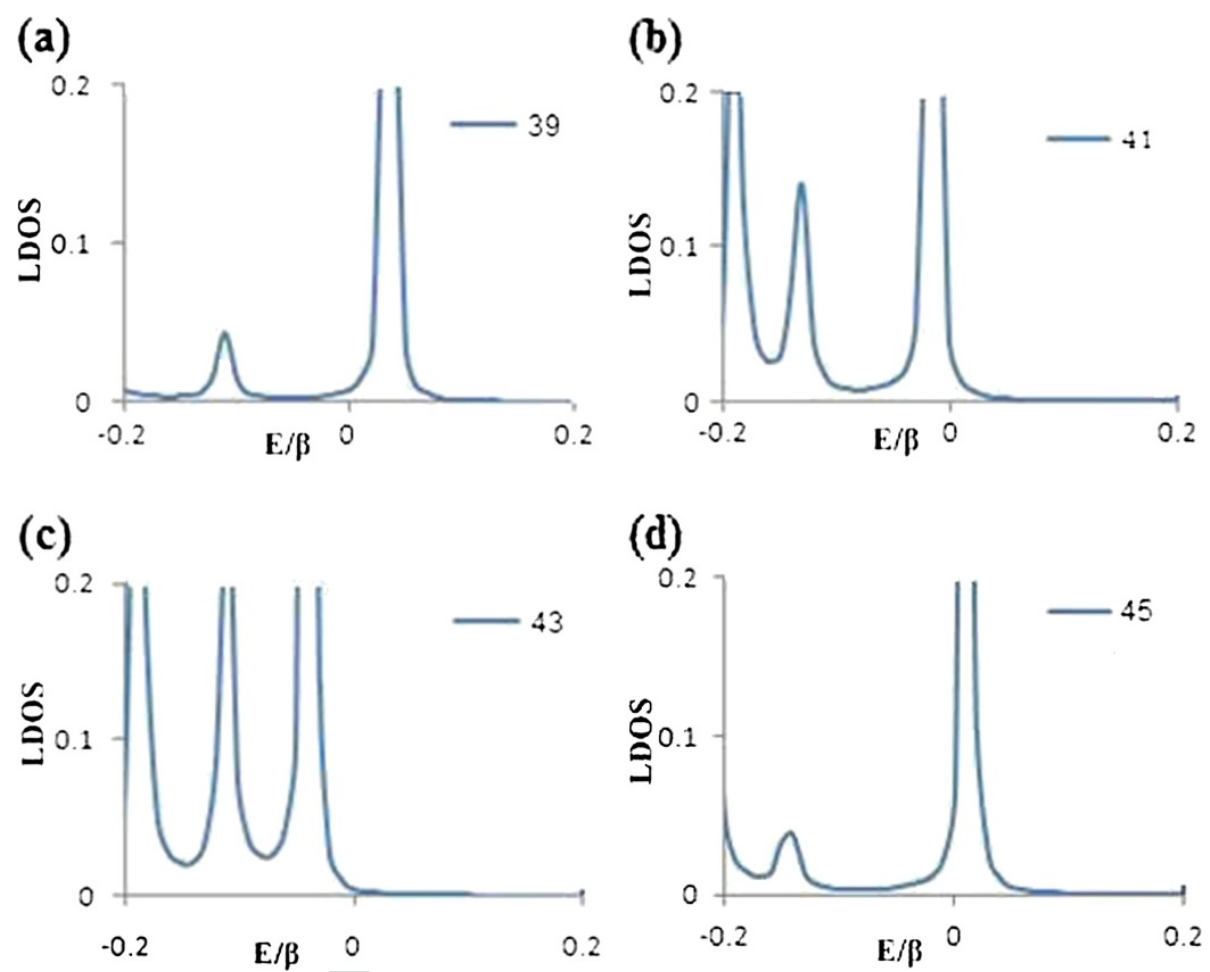

Figure 5 LDOS of sites $\mathbf{3 9}, \mathbf{4 1}, \mathbf{4 3}$, and $\mathbf{4 5}$ when one $\mathbf{H}_{\mathbf{2}}$ is adsorbed by each site. (a) LDOS of site 39 , (b) LDOS of site 41 , (c) LDOS of site 43 , and (d) LDOS of site 45.

function of the system, and $\eta$ is a positive infinitesimal energy.

$\sum_{\mathrm{L} / \mathrm{R}}$ is the left/right lead self-energies. By applying the mode-matching method and using the boundary conditions (the source is put in the left lead), these selfenergies are given by

$$
\Sigma_{\mathrm{L}}=H_{\mathrm{LD}} F_{\mathrm{L}}^{-1} ; \Sigma_{\mathrm{R}}=H_{\mathrm{DR}}^{\dagger} F_{\mathrm{R}},
$$

where they contain all information about the coupling between the leads and scattering device region (HAGNR) as well as the information about the scattering boundary condition. $H_{\mathrm{LD} / \mathrm{RD}}$ stands for coupling matrix between neighbor cells in the left/right lead and the device. Note that self-energy functions are energy-dependent and expressed in terms of the Bloch matrices $F_{\mathrm{L} / \mathrm{R}}$ where the Bloch matrix depends on the lead modes.

In the real space, $H$ is a Hamiltonian matrix where its elements are matrices with a dimension of $N_{0} \times N_{0}$. For definition of $H$ matrix elements, we used the tight-binding representation in the nearest neighbor's approximation.

We consider the hopping between the nearest neighbor atoms in the device equal to $2.75 \mathrm{eV}$ and calculate other hopping and on-site energies with respect to this value.

Using the Landaur approach, we apply a weak bias voltage between the left and right regions in the transport direction. In addition, we ignored phonons effects, and we fixed temperature at room temperature, $T=300 \mathrm{~K}$.

The total current using scattering formalism is given as follows: [15]

$$
I=\left(\frac{2 \pi e}{\eta}\right) \int T(E)\left\{f_{\mathrm{L}}(E)-f_{\mathrm{R}}(E)\right\} d E
$$

where $f_{\mathrm{L} / \mathrm{R}}=1 /\left(1+\exp \left(\left[E-\mu_{\mathrm{L} / \mathrm{R}}\right] / k_{\mathrm{B}} T\right)\right)$ is the Fermi function, and $\mu$ is the chemical potential. Note that the chemical potential of the two leads varies by the applied bias voltage, $V$, as $\mu_{\mathrm{L}}-\mu_{\mathrm{R}}=e V$ (source is at the left lead). We assumed that before applying bias voltage, the chemical potential of the whole system is $-6 \beta$ where $\beta$ is the hopping energy in the device. After applying bias voltage, all atoms in the left lead cells have the same chemical potential $\mu_{\mathrm{L}}$, while the atoms in the right lead have the same chemical potential $\mu_{R}$. However, in the device region, the chemical potential varies linearly with respect to the distance from the left edge of the 
(a)

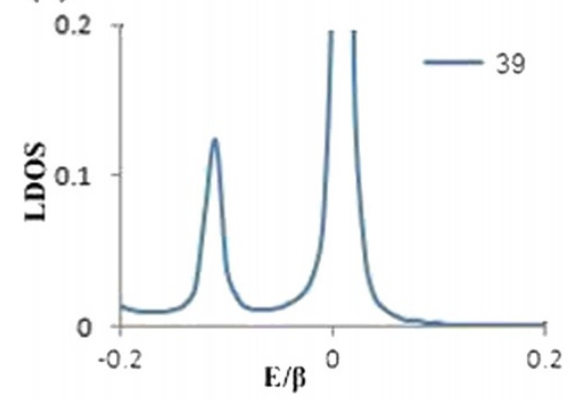

(c)

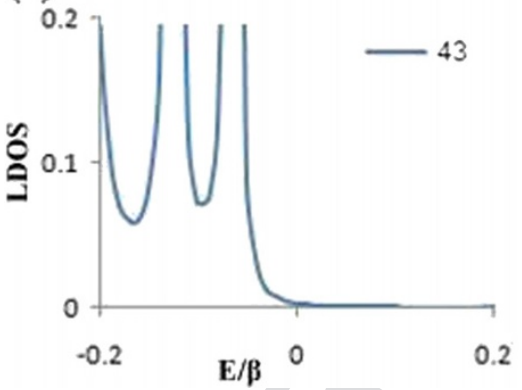

(b)

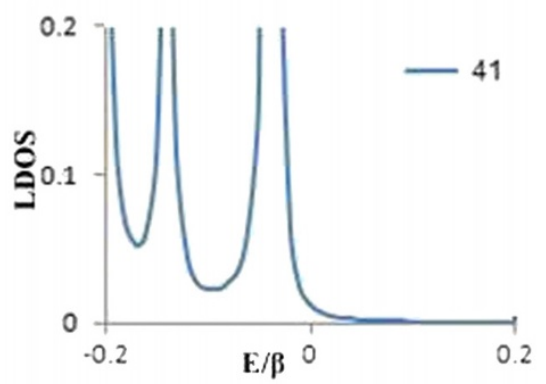

(d)

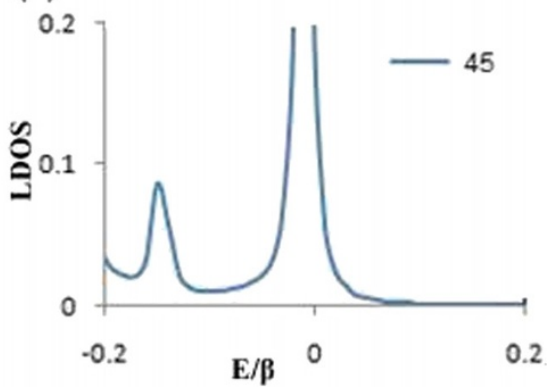

Figure 6 LDOS of sites $\mathbf{3 9}, \mathbf{4 1}, \mathbf{4 3}$, and $\mathbf{4 5}$ when one $\mathbf{O}_{\mathbf{2}}$ is adsorbed by each site. (a) LDOS of site 39, (b) LDOS of site 41, (c) LDOS of site 43 , and (d) LDOS of site 45 .

(a)

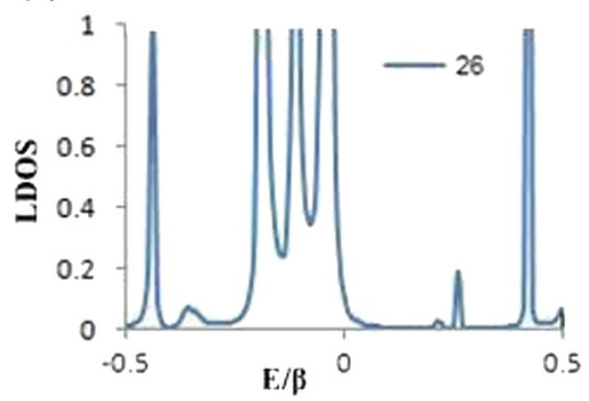

(b)

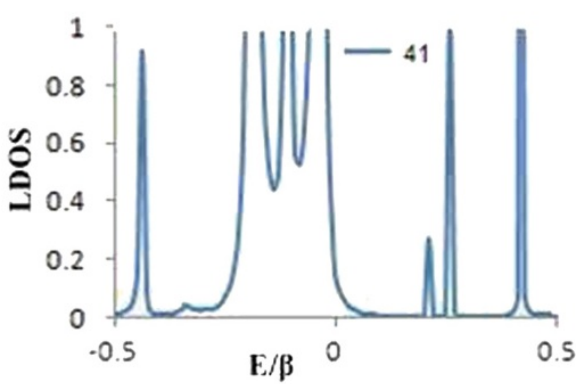

(c)

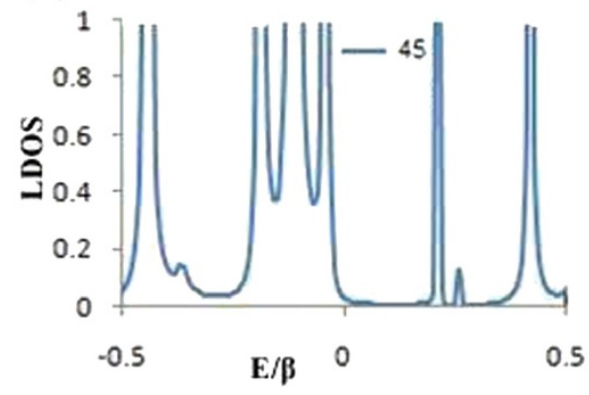

Figure $\mathbf{7}$ LDOS of sites $\mathbf{2 6}, \mathbf{4 1}$, and $\mathbf{4 5}$ when site $\mathbf{4 3}$ adsorbed a $\mathbf{H}_{\mathbf{2}}$ molecule. (a) LDOS of site 26, (b) LDOS of site 41 , and (c) LDOS of site 45. 


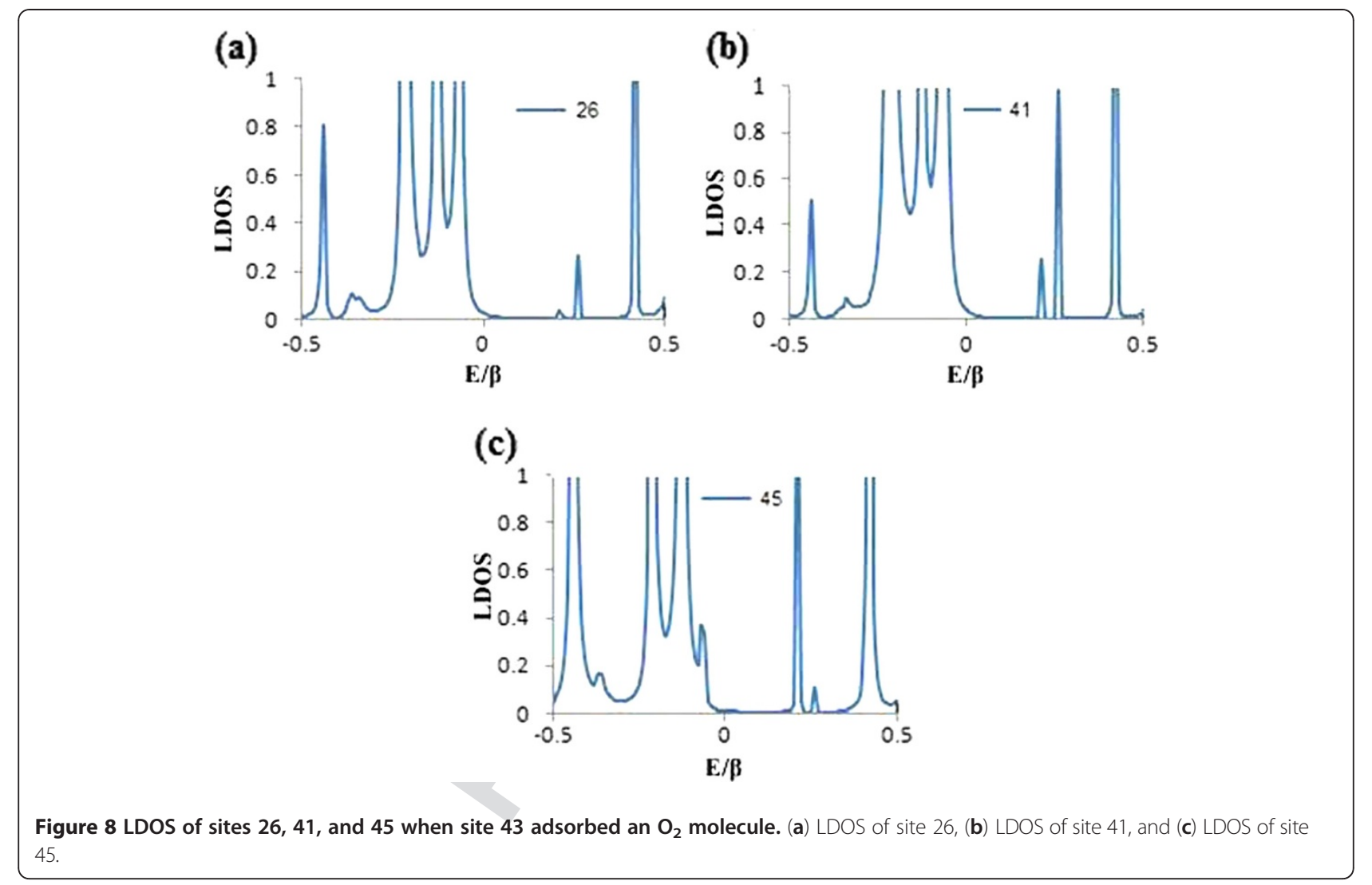

device, i.e., along the transport direction (as shown in Figure 1).

Our clean system is a HAGNR which included 90 atoms (70 carbon and 20 hydrogen atoms). Now, we consider hydrogen and oxygen molecule adsorption on the top of this system. We assumed that the leads are two-dimensional semi-infinite periodic wires of copper with body centered square lattices where the widths of all regions are the same. In the next section, we present our results. (a)

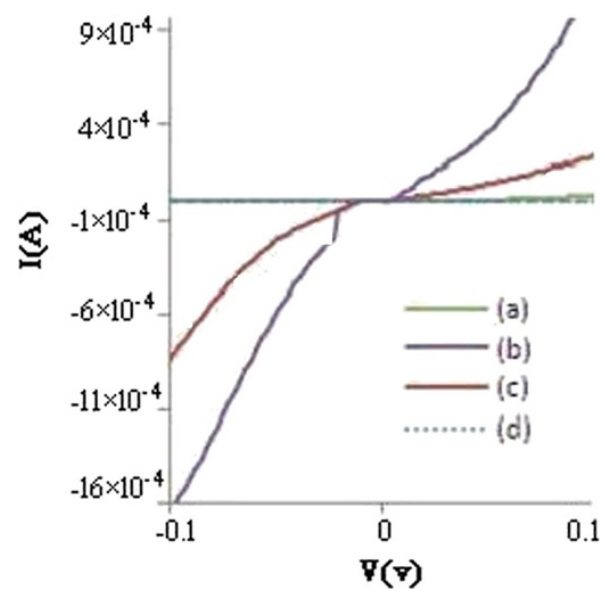

(b)

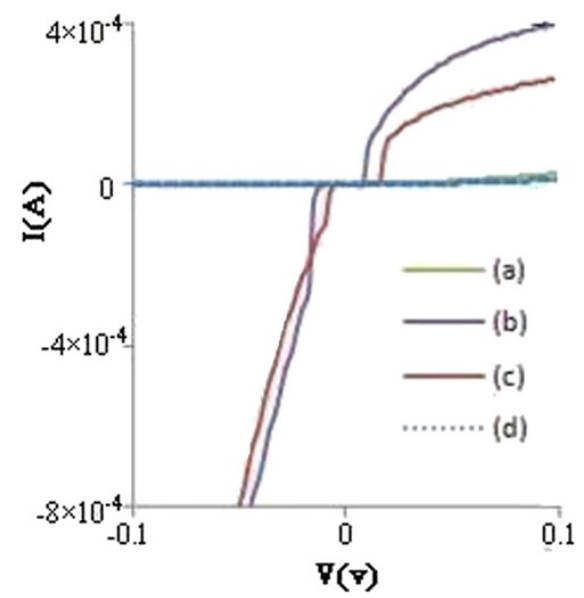

Figure $9 \mathrm{I}-\mathrm{V}$ curves of HAGNR. I-V curves of HAGNR when three (a) $\mathrm{H}_{2}$ or three (b) $\mathrm{O}_{2}$ molecules are adsorbed by (a) sites 21, 39, and 57; (b) sites 23,41, and 59; (c) sites 25,43, and 61; and (d) sites 27,45, and 63 along its length 
(a)

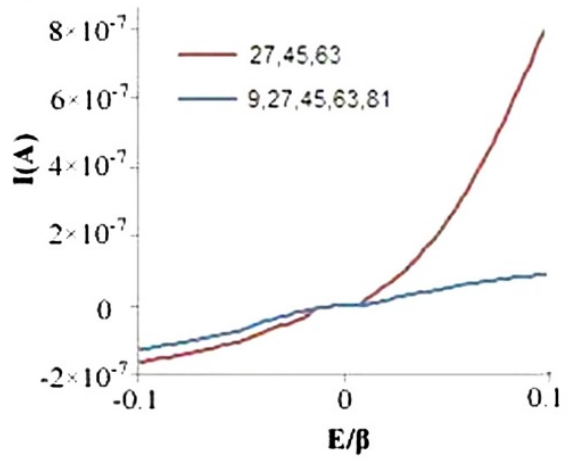

(c)

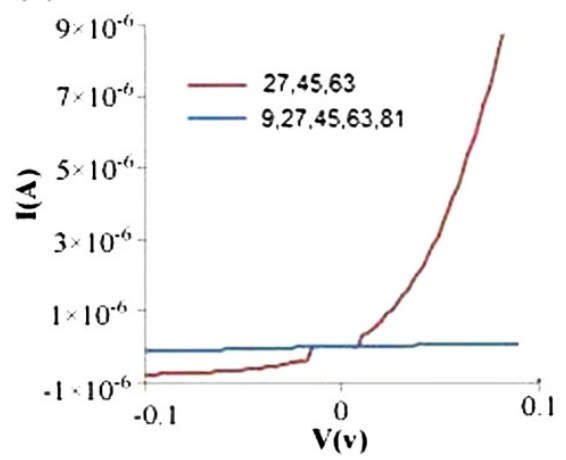

(b)

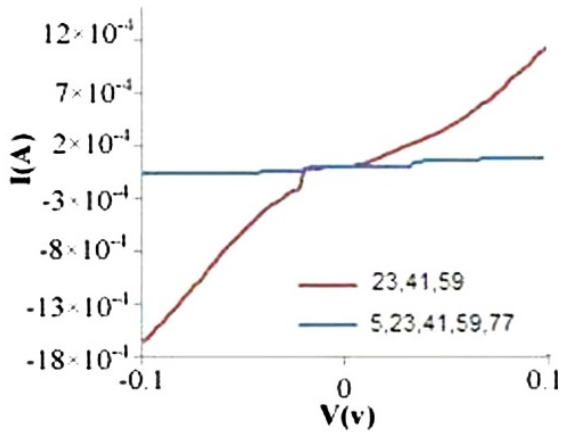

(d)

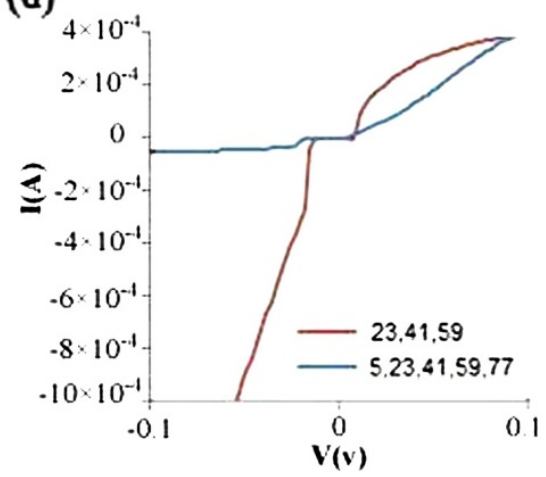

Figure 10 Comparison of $I-V$ curves. Comparison of $I-V$ curves when metallic sites 27, 45, and 63 and sites 9, 27, 45, 63, and 81 adsorbed (a) three and (c) five $\mathrm{H}_{2} / \mathrm{O}_{2}$ molecules, respectively. Semiconducting sites 23 , 41, and 59 and sites 5, 23, 41, 59, and 77 adsorbed (b) three and (d) five $\mathrm{H}_{2} / \mathrm{O}_{2}$ molecules, respectively.

\section{Result and discussion}

To see effects of hydrogen/oxygen molecule adsorption on the electronic properties of HAGNRs, first, we investigate electronic properties of our clean HAGNR system. Figure 2a,b,c,d illustrates the comparison of LDOS of sites 39, 41, 43, and 45 along the width of the clean HAGNR, respectively. It is obvious that LDOS of sites 39 and 45 have a metallic behavior, while those of sites 41 and 43 show a semiconducting feature. Therefore, the upper (lower) edge sites along the length of the (a)

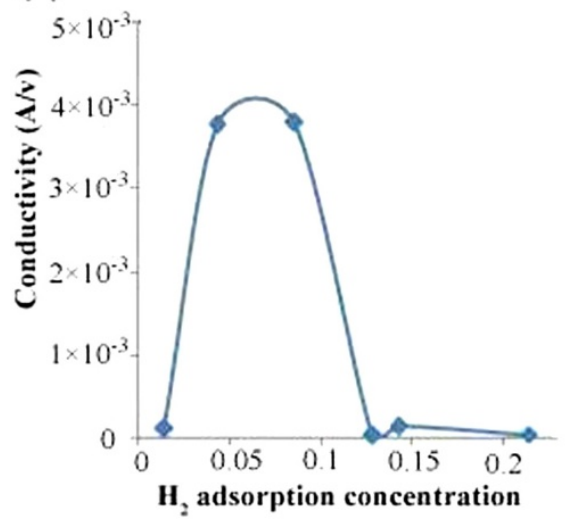

(b)

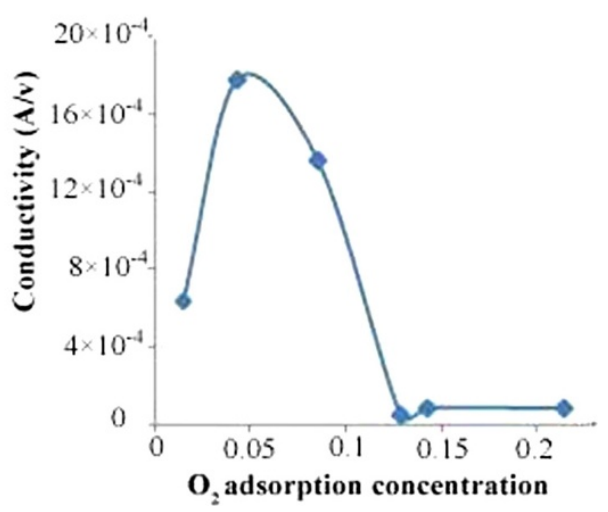

Figure 11 Conductivity of HAGNR in Figure 1 in terms of (a) $\mathrm{H}_{2}$ and (b) $\mathrm{O}_{2}$ adsorption concentration. 
HAGNR construct metallic regions separated by semiconducting regions.

Figure 3a shows the metallic and semiconducting regions for the lower edge nanowire. The middle nanowire is constructed from metallic and semiconducting regions of double-site atoms along the length of the HAGNR. Figure 3b shows this nanowire. Hence, at low adsorptions, adsorption of a hydrogen/oxygen molecule by a metallic or semiconducting carbon atom of the HAGNR could change the electronic properties of the HAGNR in different ways. Adsorptions of $\mathrm{H}_{2} / \mathrm{O}_{2}$ by metallic sites lead to a reduction of current, while those by semiconducting sites lead to an increase in current. Figure $4 \mathrm{a}, \mathrm{b}$ shows the current-voltage characteristics of our system when one hydrogen/oxygen molecule is adsorbed by sites $39,41,43$, and 45 along the width of the HAGNR. To explain this result, we should look at the LDOS of these sites for the adsorbed system. Figures $5 \mathrm{a}, \mathrm{b}, \mathrm{c}, \mathrm{d}$ and $6 \mathrm{a}, \mathrm{b}, \mathrm{c}, \mathrm{d}$ show LDOS of sites 39, 41,43 , and 45 when one $\mathrm{H}_{2} / \mathrm{O}_{2}$ is adsorbed by sites $39,41,43$, or 45 . These figures show that adsorption of $\mathrm{H}_{2} / \mathrm{O}_{2}$ by semiconducting sites converted these sites to metallic sites. Hence, this adsorption increases the current, while adsorption of $\mathrm{H}_{2} / \mathrm{O}_{2}$ by metallic sites reduces the current. To clarify this result, we plotted LDOS of the neighbor sites of adsorption site 43. Figures $7 \mathrm{a}, \mathrm{b}, \mathrm{c}$ and $8 \mathrm{a}, \mathrm{b}, \mathrm{c}$ show LDOS of sites 26,41 , and 45 , respectively, when one $\mathrm{H}_{2} / \mathrm{O}_{2}$ is adsorbed by site 43 . The semiconducting sites 26 and 41 are converted to metallic sites. In the next stage, we investigate adsorption concentration effects on the $I-V$ curve of the HAGNR. Figure 9a,b shows $I-V$ curves of the HAGNR when three $\mathrm{H}_{2}$ or three $\mathrm{O}_{2}$ molecules are adsorbed by (a) sites 21, 39, and 57; (b) sites 23, 41, and 59; (c) sites 25, 43, and 61; and (d) sites 27, 45 , and 63 along its length, respectively. We found that at high adsorption concentrations, adsorption by semiconducting sites with bigger energy gap increases current more than smaller energy gap semiconductor sites, and adsorption by more metallic sites decreases current with respect to those sites with low metallic features. Figure 10 illustrates the comparison of $I-V$ curves in which metallic sites 27,45 , and 63 adsorbed three $\mathrm{H}_{2} / \mathrm{O}_{2}$ molecules (Figure 10a); sites $9,27,45,63$, and 81 adsorbed five $\mathrm{H}_{2} / \mathrm{O}_{2}$ molecules (Figure 10c); semiconducting sites 23, 41, and 59 adsorbed three $\mathrm{H}_{2} / \mathrm{O}_{2}$ molecules (Figure 10b); and sites 5, 23, 41, 59, and 77 adsorbed five $\mathrm{H}_{2} / \mathrm{O}_{2}$ molecules (Figure 10d). We found that by increasing adsorption concentration, current decreases. To complete our results, we calculated the conductivity of the system in terms of $\mathrm{H}_{2} / \mathrm{O}_{2}$ adsorption concentration. Figure 11a,b illustrates conductivity in terms of $\mathrm{H}_{2} / \mathrm{O}_{2}$ concentration, respectively. Since at high adsorptions these systems have metallic features, so for high concentrations, we expect such reductions.

\section{Conclusions}

Using the NEGF (non equilibrium Green's function) method in the tight-binding approach, effects of gas adsorption on the electronic properties of hydrogenated armchair graphene nanoribbons are investigated. We found that at a low adsorption concentration, adsorption by metallic HAGNR sites decreases current, while adsorption by semiconductor HAGNR sites increases current in the current-voltage curve of these systems. At high adsorption concentrations, the whole system becomes metallic, so by increasing adsorption concentrations, current always decreases. Also, we found that at low adsorption, the conductivity of the system increases by increasing adsorption, while at high adsorptions, the conductivity of the system decreases by increasing adsorption. These results could be used to make a gas nanosensor.

\section{Competing interests}

The authors declare that they have no competing interests.

\section{Authors' contributions}

The authors' contributions in this article are the same. All authors read and approved the final manuscript.

\section{Author details}

${ }^{1}$ Physics Department, Faculty of Science, Razi University, Kermanshah 67149-67346, Iran. ${ }^{2}$ Nano Technology Research Laboratory, Razi University, Kermanshah 67149-67346, Iran. ${ }^{3}$ Computational Physical Science Research Laboratory, Department of Nano-Science, Institute for Studies in Theoretical Physics and Mathematics (IPM), Tehran

19395-5531, Iran.

Received: 5 January 2011 Accepted: 20 April 2012

Published: 20 April 2012

\section{References}

1. Anantram, MP, Leonard, F: Physics of carbon nanotube electronic devices. Rep. Prog. Phys. 69, 507 (2006)

2. Lu, W, Lieber, CM: Semiconductor nanowires. J. Phys. D: Appl. Phys. 39, R387-R406 (2006)

3. Wang, H, Wang, X, Li, X, Dai, H: Chemical self-assembly of graphene sheets. Nano. Res. 2, 336-342 (2009)

4. Moradian, R, Mohammadi, Y: Finite-concentration gas molecule adsorption on carbon nanotubes investigated by a tight-binding approach. Phys. Rev. B. 76, 155432 (2007)

5. Moradian, R, Mohammadi, Y, Ghobadi, N: Investigation of gas sensing properties of armchair graphene nanoribbons. J. Phys. Condens. Matter 20 425211 (2008)

6. Panella, B, Hirscher, M, Roth, S: Hydrogen adsorption in different carbon nanostructures. Carbon 43, 2209-2214 (2005)

7. Sevinçli, H, Topsaka, M, Durgun, E, Ciraci, S: Electronic and magnetic properties of $3 d$ transition-metal atom adsorbed graphene and graphene nanoribbons. Phys. Rev. B. 77, 195434 (2008)

8. Giannozzi, P, Car, R, Scoles, G: Oxygen adsorption on graphite and nanotubes. J. Chem. Phys. 118, 1003 (2003)

9. Schimmel, HG, Kearley, GJ, Nijkamp, MG, Visser, CT, de Jong, KP, Mulder, FM: Hydrogen adsorption in carbon nanostructures: comparison of nanotubes, fibers, and coals. Chem. Eur. J. 9, 4764-4770 (2003)

10. Schimmel, HG, Nijkamp, MG, Kearley, GJ, Rivera, A, de Jong, KP, Mulder, FM: Hydrogen adsorption in carbon nanostructures compared. Materials Science and Engineering B 108, 124 (2004)

11. Zhu, Z, Lu, GQ, Finnerty, J, Yang, RT: Electronic structure methods applied to gas-carbon reactions. Carbon 41, 635 (2003) 
12. Ataca, C, Aktürk, E, Ciraci, S: Hydrogen storage of calcium atoms adsorbed on graphene: first-principles plane wave calculations. Phys. Review B. 79 041406 (2009)

13. Jagiello, J, Thommes, M: Comparison of DFT characterization methods based on $\mathrm{N}_{2}, \mathrm{Ar}_{1} \mathrm{CO}_{2}$, and $\mathrm{H}_{2}$ adsorption applied to carbons with various pore size distributions. Carbon 42, 1227-1232 (2004)

14. Moradian, R, Zereshki, P, Haseli, S, Hayati, M: Investigation of electronic transport through graphene nanoribbon quantum dots. Physica E 41, 801 (2009)

15. Ferry, DK, Goodnick, SM: Transport in Nanostructures. Cambridge University Press, Cambridge (1997)

16. Datta, S: Electronic Transport in Mesoscopic Systems. Cambridge University Press, Cambridge (1997)

17. Castro Neto, AH, Guinea, F, Peres, NMR, Novoselov, KS, Geim, AK: The electronic properties of grapheme. Rev. Mod. Phys. 81, 109 (2009)

18. Koshelkin, AV: Two-particle Green's functions in non-equilibrium matter. Physics Letters B. 471, 202-207 (1999)

19. Xue, Y, Datta, S, Ratner, MA: First-principles based matrix Green's function approach to molecular electronic devices: general formalism. Chem. Phys. 281, 151-170 (2002)

20. Son, YW, Cohen, ML, Louie, SG: Energy gaps in graphene nanoribbons. Phys. Rev. Lett. 97, 216803 (2006)

21. White, CT, Li, J, Gunlycke, D, Mintmire, JW: Hidden one-electron interactions in carbon nanotubes revealed in graphene nanostrips. Nano Lett. 7, 825-830 (2007)

22. Brey, L, Fertig, HA: Electronic states of graphene nanoribbons studied with the Dirac equation. Phys. Rev. B. 73, 235411 (2006)

23. Ezawa, M: Peculiar width dependence of the electronic properties of carbon nanoribbons. Phys. Rev. B. 73, 045432 (2006)

doi:10.1186/2228-5326-2-3

Cite this article as: Moradian and Nazeri: Retracted: Electrical conductivity of hydrogenated armchair nanoribbon as a gas sensor using non-equilibrium Green's function method. International Nano Letters 2012 2:3.

\section{Submit your manuscript to a SpringerOpen ${ }^{\circ}$ journal and benefit from:}

- Convenient online submission

- Rigorous peer review

- Immediate publication on acceptance

- Open access: articles freely available online

- High visibility within the field

- Retaining the copyright to your article

Submit your next manuscript at $\gg$ springeropen.com 